\title{
A model for distribution centers location-routing problem on a multimodal transportation network with a meta-heuristic solving approach
}

\author{
Saeed Fazayeli $^{1} \cdot$ Alireza Eydi $^{1} \cdot$ Isa Nakhai Kamalabadi $^{1}$
}

Received: 16 November 2016/ Accepted: 26 June 2017/Published online: 1 July 2017

(C) The Author(s) 2017. This article is an open access publication

\begin{abstract}
Nowadays, organizations have to compete with different competitors in regional, national and international levels, so they have to improve their competition capabilities to survive against competitors. Undertaking activities on a global scale requires a proper distribution system which could take advantages of different transportation modes. Accordingly, the present paper addresses a location-routing problem on multimodal transportation network. The introduced problem follows four objectives simultaneously which form main contribution of the paper; determining multimodal routes between supplier and distribution centers, locating mode changing facilities, locating distribution centers, and determining product delivery tours from the distribution centers to retailers. An integer linear programming is presented for the problem, and a genetic algorithm with a new chromosome structure proposed to solve the problem. Proposed chromosome structure consists of two different parts for multimodal transportation and location-routing parts of the model. Based on published data in the literature, two numerical cases with different sizes generated and solved. Also, different cost scenarios designed to better analyze model and algorithm performance. Results show that algorithm can effectively solve large-size problems within a reasonable time which GAMS software failed to reach an optimal solution even within much longer times.
\end{abstract}

Keywords Location-routing problem $\cdot$ Multimodal transportation · Distribution center - Genetic algorithm

Alireza Eydi

Alireza.eydi@uok.ac.ir

1 Department of Engineering, University of Kurdistan, Pasdaran Blvd., Post Box No.: 416, Sanandaj, Iran

\section{Introduction}

Effective and efficient transportation of materials and products through the chain of suppliers, manufacturers, assemblers, distribution centers (DCs), retail stores, and customers is crucial in the competitive world of today. In other words, transportation is a key part of a supply chain which ensures on-time delivery of raw materials and finished products (Crainic 2003a). Decisions made regarding a supply chain transportation system can be classified into three different levels. The first level refers to strategic decisions with long-term (several years) effects on the supply chain. These are mainly the decisions made on the transportation system design and supplying resources (facility location, size and capacity of facilities, facility and plant layout, transportation fleet). The next level is tactical decisions which has mid-term effects (several months or quarters) and include production and distribution planning and resource allocation issues (facility space, fleet size and shipments packaging strategies). Third level is operational decisions which are made on a daily or weekly basis. Orders aggregation, shipments, and vehicle fleet dispatch are examples of these decisions (Daskin et al. 2005). For the last four decades, researchers have studied combination of these decisions with a combinational view to supply chain and transportation problems. In this regard, locationrouting problem (LRP) considers both location (strategic level) and routing (tactical level) problems. LRP represents a relatively new form of location problems addressing locating facilities such as distribution centers and depots, where routing aspects are simultaneously considered. Reflecting interactions between facility location and fleet routing, LRP can provide a better view for logistics analysts and managers, preventing poor and local optimizations. LRP had growing trends especially in recent years. 
Govindan et al. (Govindan et al. 2014) used LRP in perishable products distribution system design. RiquelmeRodríguez et al. (2016) present and compare two methods for locating water depots along the road network and used arc routing in their problem. And Gao et al. (2016) introduced ant colony optimization with clustering for solving the dynamic location-routing problem. Seyedhosseini et al. (2016) reviewed dynamic location problems based on their models, solution, methods and applicability and analyzed gaps for future research. It can also be used for dynamic LRPs.

In general, because of geographical distances between producers and consumers demand for goods transportation has raised (Chopra and Meindl 2001). Transportation, especially in long distances, is a world class business which cannot be accomplished through roads only due to availability and cost concerns. So there is a need for other transportation modes or a combination of them (i.e., multimodal transportation). European Conference of Ministers of Transport in 2001 defined multimodal transportation as "movement of goods in one and the same loading unit or vehicle, which uses successively two or more modes of transport without handling the goods themselves in changing modes". Multimodal transportation has experienced an increased importance during recent years and now multimodal transportation is competing with single-mode transportation. In this regard, many transportation companies have established multimodal transportation services. The second point is that, multimodal transportation is becoming an important policy for organizations because of its advantages in terms of cost and coordination between modes in large-scale cargoes (OECD 1997). The third point is that, in references and handbooks of transportation, multimodal transportation is treated as an independent and well-separated transportation mode (Button 1994). Last but not least, since 1990, the number of papers on multimodal transportation follows a growing trend (Bontekoning et al. 2004). Some researchers have reviewed the related literatures (SteadieSeifi et al. 2014). To sum up, multimodal transportation is a relatively new field of research, with its increasing importance during recent years.

The objective of this study is to help designing a distribution system with two transportation network types. First network transfers products from supplier (with determined location) to DCs via a multimodal transportation system. On second network, products are distributed among customers via routing tours started from DCs on a single-mode network. In this problem, different questions will be answered: which multimodal route should be used to transfer products to DCs? Is there need to change mode in determined multimodal routes? And if yes, mode changing facilities should locate on which multimodal terminals? Each routing tour generated from open DCs, meet which customers and in what order? In fact, this study considers LRP and multimodal route selection problem simultaneously.

Research methodology is consists of the following sections. First, introduced problem modeled as a linear programming with a cost minimizing objective. Then mathematical model solved with GAMS optimizing software and CPLEX solver for two different numerical instances. Also, a genetic algorithm generated for the problem and its results compared with CPLEX results.

The following section gives a review on related literatures on multimodal logistics and location-routing problem. Then, the subsequent section gives an explanation of the proposed problem, followed by the section introducing the corresponding mathematical model. In the following section, the used solving approach is introduced before demonstrating and solving two different numerical cases, where the cases are further analytical discussed. The final section concludes the paper.

\section{Literature review}

This study integrates multimodal transportation and LRP. Both of them are apparently independent fields of research and will be discussed separately. Also a few papers jointly discussing the two concepts are also cited. Different researchers used combination of problems to define their model. Shen et al. (2003) presented location and inventory problems together. Azadeh et al. (2017) considered vehicle routing and inventory decisions simultaneously. Beginning of LRP development can be traced back to 70 and $80 \mathrm{~s}$ when Laporte and Nobert (1981) presented a single-depot model which was subsequently used by other researchers. Loperte et al. (1983) considered multi-depot LRP to further develop the problem. Thereafter, various authors has introduced different approaches to the problem which can be classified based on problem structure (single-echelon or multi-echelon), type of data (deterministic, probabilistic, or fuzzy), number of product types (single-product or multiproduct), number and capacity of facilities (single-facility or multi-facility, capacitated or incapacitated), type and capacity of vehicles (homogenous or heterogeneous, capacitated or incapacitated), time window and problem type (soft or hard), number of objective functions (singleobjective or multi-objective), and solving methods (exact, heuristic, meta-heuristic, and combinational). In this regard, some researchers (e.g., Nagi and Salhi 2007; Prodhon and Prins 2014; Lopes et al. 2013; Drexl and Schneider 2014) present review papers.

$\mathrm{Wu}$ et al. (2017) designed a three echelon LRP. They also considered tight time windows and time deadlines to create services for high-speed trains. Albareda-Sambola 
et al. (2012) proposed a multi-period LRP model and a stable model against time. An approximation based on replacing vehicle routes by spanning trees is proposed, and its capability for providing good quality solutions is assessed in a series of computational experiments Karaoglan et al. (2012) used goods pick and delivery planning in LRP. The authors proposed two polynomial-size mixed integer linear programming formulations for the problem and a family of valid inequalities to strengthen their formulation. Rodriguez-Martin and Salzar-Gonzalez (2014) considered locations of and routing through hub facilities. Proposed model decide on the location of hubs, the allocation of nodes to hubs, and the routing among the nodes allocated to the same hubs, with the aim of minimizing the total transportation cost. Ahmadi-Javid and Seddighi (2013) set probabilistic facility capacity and disruption risk in the problem. The goal is to determine the location, allocation and routing decisions that minimize the annual cost of location, routing and disruption. TavakkoliMoghaddam and Raziei (2016) took demands as fuzzy numbers. They present a bi-objective location-routing-inventory problem with heterogeneous fleets in a two-echelon distribution network and fuzzy demands. Ghezavati and Beigi (2016) proposed a bi-objective mathematical model for location-routing problem in a multi-echelon reverse logistic network. Their proposed network consists of hybrid collection/inspection centers, recovery centers and disposal centers. They considered total cost minimization and minimizing the maximum time of completion of the collecting return products as objective functions. Hiassat et al. (2017) also considered location-routing-inventory problem for perishable products distribution. Samanlioglu (2013) developed a LRP for dangerous materials handling. In this paper, a new multi-objective location-routing model is developed, and implemented in the Marmara region of Turkey. Shahabi et al. (2014) added inventory management to three levels LRP. They also assumed that demand across the retailers is to be correlated as Najjartabar et al. (2016) which considered correlated demands in location-inventory problem in a three level supply chain. Aghighi and Malmir (2016) used location-routing inventory problem on perishable product distribution system design. In this paper, authors considered stochastic demands and travel times and solved problem in two phases. And Fazel Zarandi et al. (2013) considered time window, fuzzy demand, and fuzzy travel times at the same time. Moreover these papers, Govindan et al. (2014) proposed LRP in a sustainable network. Sustainable networks have a growing trend in supply chain problems. Afshar-Bakeshloo et al. (2016) developed a model, named Satisfactory-Green Vehicle Routing Problem. It consists of routing a heterogeneous fleet of vehicles to serve a set of customers within predefined time windows. Also, Najjartabar-Bisheh et al. (2017) analyzed the role of third-party companies in a sustainable supply chain design.

As LRPs, multimodal transportation papers can be classified based on their planning time horizon (strategic, tactical, or operational). More recently, Crainic (2003) and Steadie Seifi et al. (2014) classified the researches on multimodal transportation in two review papers and can be referred for more studies.

Following studies are joint papers for both LRP and multimodal transportation. Li et al. (2007) introduced location of terminals problem on a multimodal transportation network. The proposed model simultaneously considers choices of travelers on route, parking location and mode between auto and transit. Chiadamrong and Kawtummachai (2008) designed a methodology to support decision making on sugar industry. This aim of this paper is to suggest the best inventory position and transportation route in the distribution system considering different transportation options. Tiwari et al. (2013) considered route selection on a multimodal transportation network with several objectives: minimization of travel time and travel cost, later schedules and delivery times of every service provider in each pair of location, and lastly variable cost must be included in every location. Alumur et al. (2012) considered hub location problem and hub network design and assumed the hub and non-hub nodes are linked via multimodal transportation. Also, Moccia et al. (2010) considered hub facilities on multimodal network, but they proposed and solved a routing problem. Xie et al. (2012) considered mode changing facility location and multimodal route selection problem on a multimodal network for dangerous materials transportation. They considered different origin/destination pairs and selected the best multimodal route with determining mode changing points. Hajibabai and Ouyang (2013) presented a location and routing problem for biofuel transformation facilities on a multimodal network. In this study, first, some locations were selected for biofuel transformation facilities which were to be located between supplier and customers; then, multimodal routes were determined connecting suppliers to the facilities and then the facilities to customers. However, they presumed the routes to be of multimodal nature, neglecting to consider different modes and mode changing issues. Tuzkaya et al. (2014) presented distribution facilities location problem in a multimodal transportation network. In their approach, in the first phase, using analytic network process (ANP), a decision was made on the best transportation mode and the best potential sites to establish facilities; in the second phase, distribution facilities location problem was solved. Finally, Ayar and Yaman (2012) added time windows to multimodal routing problems.

Even though location-routing problem has experienced various developments during recent past, yet few papers 
are reported, wherein multimodal transportation is accounted for in such problems. To the best of our knowledge, no paper in the literature considered products delivery tours from DCs to customers while determining routs from supplier to DCs on multimodal network at the same time. Using multimodal logistics is an indispensable option for world class organizations, so the present paper is an attempt to fill-in this gap in the research field of location-routing.

\section{Problem description}

In a location-routing problem, a supplier of a product seeks to satisfy demands raised by some retailers in different locations. To do this, the supplier has to establish a number of DCs. The problem is defined as the determination of locations for DCs. The transportation network connecting supplier to potential DCs supposed to be a multimodal network including three transportation modes (road, railways and seaways); and transportation from DCs to retailers is performed via a road network (one mode). On road network products pass through routing tours to be moved from DCs to retailers. Figure 1 demonstrates the problem schematically. The multimodal links on the figure can represent either of a road, a railway, a seaway, or a combination of these transportation modes with multimodal terminals to change modes.

This problem is based on the following assumptions:

- Each transportation mode has a specific and determined cost.

- During the transportation operation, transportation mode may be changed at some nodes along the multimodal network.

Fig. 1 Schematic of the proposed problem
- Transportation mode can be changed at multimodal terminals where at least two different transportation modes start/end. Providing mode change facilities imposes a fixed cost to the system.

- Product unit does not change when transportation mode changes.

- DCs need a fixed cost to establish.

- Capacities of DCs are constrained by the capacities of the allocated vehicles.

- Retailers' demands are determined and satisfied through routing tours starting from established DCs. The tours return back to the DC after meeting a number of retailers.

- For each DC, one vehicle is allocated with one tour determined for each vehicle.

- Each retailer is assigned to a pair of vehicle and DC.

- Total allocated retailers' demand cannot exceed the vehicle capacity.

\section{Problem formulation}

The following notations are used to describe the problem.

Sets

\begin{tabular}{|c|c|}
\hline $\mathrm{O}$ & Supplier location \\
\hline M & $\begin{array}{l}\text { Transportation modes, } \\
m \in\{H(\text { road }), R(\text { rail }), S(\text { sea })\}\end{array}$ \\
\hline$V_{\text {one }}$ & $\begin{array}{l}\text { Set of intermediate nodes (between supplier } \\
\text { and DCs) crossed by only one transportation } \\
\text { mode }\end{array}$ \\
\hline$V_{\mathrm{HR}}$ & $\begin{array}{l}\text { Set of intermediate nodes (between supplier } \\
\text { and DCs) crossed by road and railway } \\
\text { transportation modes only }\end{array}$ \\
\hline
\end{tabular}

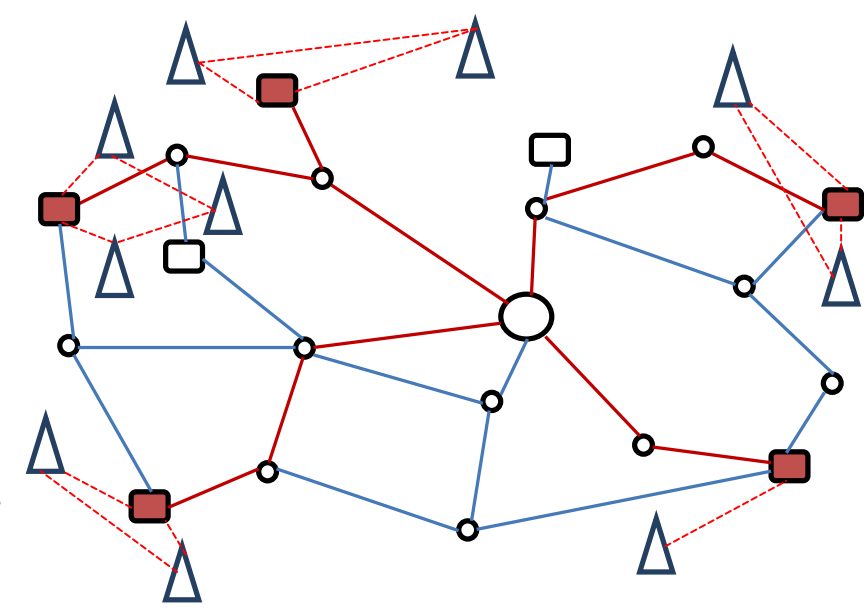


$V_{\mathrm{HS}} \quad$ Set of intermediate nodes (between supplier and DCs) crossed by road and seaway transportation modes only

$V_{\mathrm{RS}} \quad$ Set of intermediate nodes (between supplier and DCs) crossed by railway and seaway transportation modes only

$V_{\text {two }} \quad$ Set of intermediate nodes (between supplier and DCs) crossed by more than one transportation modes, $V_{\text {two }}=\left\{V_{\mathrm{HR}} \cup V_{\mathrm{HS}} \cup V_{\mathrm{RS}}\right\}$

$V \quad$ Set of nodes including supplier node as well as the nodes between supplier and potential DCs, $V=\left\{V_{\text {one }} \cup V_{\text {two }} \cup O \cup J\right\}$

$E_{m} \quad$ Set of arcs for each transportation mode from supplier to DCs, $E_{m}, m \in\{H, R, S\}$

$J \quad$ Set of potential DCs

I Set of retailers

$G=I \cup J \quad$ Set of potential DCs and retailers

$K \quad$ Set of vehicles to be used to deliver products from DCs to retailers

Parameters

$d_{i j} \quad$ Distance between nodes $i$ and $j, i, j \in G$

$D_{a b} \quad$ Distance between nodes $a$ and $b, a, b \in V \cup J$

$q_{i} \quad$ Raised demand by retailer $i$

$Q_{k} \quad$ Capacity of vehicle $k$

$C_{k} \quad$ Unit product distribution cost per unit distance from DCs to retailers by vehicle $k$

$C_{m} \quad$ Unit product transportation cost per unit distance for each mode

$C_{V} \quad$ Fixed cost of providing a mode changing facility at node $v, v \in V_{\text {two }}$

$F_{j} \quad$ Fixed cost of establishing a DC at node $j$

$\theta \quad$ Number of retailers

$n \quad$ Number of DCs

Decision variables

$z_{j}= \begin{cases}1 & \text { If a DC is established at potential node } j_{l} \\ 0 & \text { Else }\end{cases}$

$x_{i j k}= \begin{cases}1 & \text { If vehicle } k \text { goes immediately from node } i \text { to node } j, i, j \in G \\ 0 & \text { Else }\end{cases}$

$u_{i j}= \begin{cases}1 & \text { If retailer } i \text { is assigned to } \mathrm{DC} j \\ 0 & \text { Else }\end{cases}$

$y_{v}= \begin{cases}1 & \text { If transportation mode is changed at node } v \\ 0 & \text { Else }\end{cases}$

$w_{a b}^{j m}$ Amount of products passing, on the transportation mode $m$, through link $a-b$ to DC $j, a, b \in V \cup J$
Moreover, $R_{i k}$ is a slack variable used in sub-tour elimination constraint, and $B$ is large enough constant parameter. Note that, if three different modes cross over one another a node, a combination of the modes is accounted for and the node will be taken as a member of all three sets, namely $V_{\mathrm{HR}}, V_{\mathrm{HS}}$ and $V_{\mathrm{RS}}$.

The problem formulation is as follows:

$$
\begin{aligned}
\operatorname{Min} Z= & \sum_{i \in G} \sum_{j \in G} \sum_{k \in K} C_{k} d_{i j} x_{i j k}+\sum_{j \in J} F_{j} z_{j} \\
& +\sum_{(a, b) \in E_{m}} \sum_{m \in M} \sum_{j \in J} D_{a b} C_{m} w_{a b}^{j m}+\sum_{v \in V_{\mathrm{two}}} C_{V} y_{v}
\end{aligned}
$$

s.t.

$$
\begin{aligned}
& \sum_{(a, b) \in E_{m}} \sum_{m \in M} \sum_{j \in J} w_{a b}^{j m}-\sum_{(b, c) \in E_{m}} \sum_{m \in M} \sum_{j \in J} w_{b c}^{j m}=0 \\
& \forall b \in V_{\text {one }} \cup V_{\mathrm{two}} \\
& \sum_{(a, b) \in E_{m}} \sum_{j \in J} w_{a b}^{j m}-\sum_{(b, c) \in E_{m}} \sum_{j \in J} w_{b c}^{j m} \leq \mathrm{By}_{b} \\
& \forall b \in V_{\mathrm{two}}, m \in M
\end{aligned}
$$$$
\sum_{m \in M} \sum_{(a, j) \in E_{m}} w_{a j}^{j m}-\sum_{i \in I} q_{i} u_{i j}=0 \quad \forall j \in J, a \in V
$$$$
\sum_{i \in G} u_{i j}=1 \quad \forall j \in J
$$$$
w_{a b}^{j m} \leq \mathrm{Bz}_{j} \quad \forall m \in M,(a, b) \in E_{m}, j \in J
$$$$
\sum_{j \in G} \sum_{k \in K} x_{i j k}=1 \quad \forall i \in I
$$$$
\sum_{i \in I} q_{i} \sum_{j \in G} x_{i j k} \leq Q_{k} \quad \forall k \in K
$$

$\sum_{p \in G} x_{i p k}-\sum_{p \in G} x_{p i k}=0 \quad \forall k \in K, i \in G$

$\sum_{i \in I} \sum_{j \in J} x_{i j k} \leq 1 \quad \forall k \in K$

$\sum_{k \in K} x_{j r k}+z_{j}+z_{r} \leq 2 \quad \forall r=1, \ldots, n, r, j \in J$

$-u_{i j}+\sum_{p \in G}\left(x_{i p k}+x_{p j k}\right) \leq 1 \quad \forall i \in I, j \in J, k \in K$

$\sum_{i \in I} \sum_{k \in K} x_{j i k}-z_{j} \geq 0 \quad \forall j \in J$

$\sum_{i \in I} x_{j i k}-z_{j} \leq 0 \quad \forall j \in J, k \in K$

$R_{i k}-R_{r k}+\theta x_{i r k} \leq \theta-1 \quad \forall i, r \in I, k \in K$

$z_{j}, u_{i j}, y_{v} \in\{0,1\} \quad \forall i \in I, j \in J, v \in V_{\text {two }}$

$x_{i j k} \in\{0,1\} \quad \forall i, j \in G, k \in K$

$w_{a b}^{j m}, R_{i k} \geq 0 \quad \forall i \in I, k \in K, a, b \in V, j \in J, m \in M$. 
Objective function (1) seeks to minimize costs including multimodal transportation costs and location-routing costs. First and second sentences are about location-routing costs which calculate cost of routing from DCs to retailers and DCs establishment fixed cost, respectively. Third and fourth sentences, respectively, calculate cost of transportation from supplier to DC on multimodal network, and cost of providing mode changing facilities as multimodal transportation costs. Constraint set (2) defines flow conservation on multimodal network and states that product flow inters to a node should be to equal product flow exits it. Mode changing can be happened only on multimodal terminals (nodes that more than one mode inters or exits the node). For each transportation mode, if products inter to a multimodal network and leave it with the same mode, mode changing will not happen and the corresponding variable $\left(y_{v}\right)$ will be zero. Same logic uses for changing modes. Constraint set (3) illustrate this for each node $v \in V_{\mathrm{two}}$. As accounted for by constraint set (4), sum of products arriving at a DC should be equal to total demands raised by the assigned retailers to that DC. Based on the constraint set (5), each retailer is assigned to exactly one DC. Constraint set (6) ensures that no product can be dispatched to a non-established DC and if DC establishment variable $\left(z_{j}\right)$ takes zero, all the product flows ending to that DC take zero as well. Constraint set (7) assigns each retailer to exactly one vehicle, while the constraint set (8) limits the capacity of the vehicles and ensures that summation of assigned customers demand does not pass vehicle capacity. In each product delivery tour on road network, every vehicle once entered a node will definitely exit that node and constraint set (9) guarantees that. Constraint set (10) is to ensure that each and every vehicle is allocated to at most one DC. The absence of common links between DCs is promised by constraint set (11). The constraint set (12) assures that a retailer is assigned to a DC if and only if there is a route from the DC to that retailer. Constraint sets (13) and (14) state that vehicles can be dispatched only from established DCs. Constraint set (15) relates to sub-tour elimination, and constraints (16), (17), and (18) define variables. The last three constraints determine model variables signs.

\section{Solving approach}

To validate mathematical model, two different numerical cases are solved by optimization software GAMS. Also, due to linear nature of the model, CPLEX solver was further used. The numerical cases were run under different scenarios using a personal laptop equipped with an Intel ${ }^{\circledR}$ Core $^{\mathrm{TM}} \mathrm{i} 3 \mathrm{CPU}$ at $2.4 \mathrm{GHz}$ and $6 \mathrm{~GB}$ of RAM.
A location-routing problem combines location and routing problems which both of them are NP-hard class problems, so LRP is NP-hard as well (Bontekoning et al. 2004). To solve a NP-hard problem, a proper solving algorithm and method is required because as the size of the problem increases, processing time increases exponentially, as is obviously seen in the second numerical case.

In this study, a genetic algorithm with a new chromosome structure is presented to solve problems with different sizes. The proposed algorithm is composed of the following components.

\section{Chromosome presentation}

Proposed chromosome consists of two separate parts. The first part refers to the multimodal routes from supplier to DCs (on multimodal network), and the second part refers to DCs location and tours routing from DCs to retailers (location-routing problem).

(a) In the first part, a matrix is developed to represent the multimodal route. The matrix dimensions are determined by the number of potential DC and intermediate nodes (between supplier and potential DCs). Number of potential DCs is taken as the number of matrix rows and each row represents a multimodal route for the corresponding potential DC. Regarding the number of columns, for each intermediate node two columns added to the matrix, i.e., each node is represented by two elements in each row (multimodal route). The first element has a binary ( 0 or 1 ) value showing if the intended node is used in the route or not. The second element distinguishes the transportation mode via which the product travels from the current node to the next one (one for road transportation, two for rail transportation, and three for sea transportation). Also, an extra first column is inserted to show the transportation mode via which products travel from the supplier to the first intermediate node along the route. Here the subject is clarified by a numerical example. Suppose a multimodal network with one supplier, six intermediate nodes, and four potential DCs. The following matrix expresses typical routes from the supplier to DCs.

$$
\left[\begin{array}{lllllllllllll}
1 & 1 & 1 & 0 & 0 & 1 & 1 & 0 & 0 & 0 & 0 & 1 & 2 \\
2 & 0 & 0 & 1 & 2 & 0 & 0 & 1 & 2 & 1 & 2 & 0 & 0 \\
1 & 0 & 0 & 0 & 0 & 1 & 3 & 1 & 3 & 0 & 0 & 0 & 0 \\
2 & 1 & 1 & 0 & 0 & 1 & 2 & 0 & 0 & 0 & 0 & 1 & 1
\end{array}\right] .
$$

The first row represents a route from the supplier to potential DC number one (DC 1). The first element 
along the row is 1 , that is, the transportation starts from the supplier on road mode. The second element along the row is 1 , which indicates the first intermediate node along the route. The third element shows that the transportation proceeds from the node 1 via road transportation. Being zero, the next pair of elements reveals that the intermediate node 2 is not used in the route. The sixth and seventh elements along the row show that the route enters the intermediate node 3 and leaves the rout via road transportation. And finally the last two elements determine that the intermediate node 6 presents along the route, and the route continues towards potential DC 1 by rail transportation with a mode change happening at node 6 . Applying this process to the next DCs, row 2 shows that route to DC number two (DC 2) is on rail network and crosses the intermediate nodes 2, 4 and 5. Furthermore, row 3 presents the route to DC number three (DC 3 ). Nodes 3 and 4 uses in this route and transportation mode change at node 3 from road to seaway. Lastly, row 4 corresponds to the route to potential DC number four (DC 4); it indicates that intermediate nodes 1,3 and 6 are used in the route and mode changing happened from railway to road on node 1 and 6 and from road to railway on node 3 .

(b) The second part of the chromosome is a string of numbers representing established DCs and sequence of retailers along routing tours. The string length is equal to total number of retailers plus potential DCs minus one. To demonstrate the string, a sample string with nine retailers and four potential DCs is presented as follows:

\section{5 .}

The numbers 1-9 present 9 retailers, and the numbers 10,11 , and 12 refer to the DCs. Retailers are assigned to DCs via the following process. From the start of the string to one of the numbers 10,11 , or 12 (the one appeared first) will be assigned to DC 1 . Obviously, if the string was started with one of the corresponding numbers to DCs, no retailer would be assigned to DC 1 . In this case, retailers 1,2 , and 4 will be assigned to DC 1 , with the same sequence. The retailers falling between the first and second corresponding numbers to DCs $(10,11$ and 12) will be assigned to DC 2 . In this example, as number 11 comes right after number 12 , no retailer is assigned to DC 2, i.e., DC2 is not going to be established. Similarly, the retailers falling between the second and third corresponding numbers to DCs $(10,11$ and 12) will be assigned to DC 3 . In this case, retailers 8 , 9,7 , and 6 are assigned to DC 3, with the same sequence of meeting the retailers. And lastly, retailers falling within the third corresponding number to DCs $(10,11$ and 12$)$ to the end of the string (retailers 3 and 5, respectively, in this example) are assigned to DC 4 .

\section{Generation of initial population}

Two different populations with equal numbers should be generated for the two different parts of the chromosome. Each member of the matrix part of the chromosome shows a possible route from supplier to potential DCs. To generate an initial population for this part, the algorithm is fed by all inbound links into intermediate nodes along with their types. The algorithm starts from potential DC 1 and selects a random inbound link from the set of corresponding inbound links. Then starting node of the selected link is determined before a random inbound link is assigned to the nodes. This process continues until the node 0 (supplier) is reached. The obtained route is then transformed into the corresponding chromosome representation as described above. Repeating the process for other potential DCs, matrix rows and columns are formed.

The initial population for the string part of chromosome is created by a routine called "randperm" in MATLAB. For each matrix part of the chromosome, a string part is generated and their costs are summed up into a single response.

\section{Parents selection mechanism}

Parent selection mechanism is an important part of the genetic algorithm. In the present research, roulette wheel selection mechanism was used.

\section{Crossover operator}

In crossover operation, for the matrix part, in selected parents rows are replaced with each other, as follows: 
First parent

$\left[\begin{array}{lllllllllll}1 & 1 & 1 & 0 & 0 & 1 & 1 & 0 & 0 & 1 & 2 \\ 2 & 1 & 1 & 1 & 3 & 0 & 0 & 1 & 3 & 0 & 0 \\ 2 & 0 & 0 & 0 & 0 & 1 & 1 & 1 & 1 & 1 & 2 \\ 2 & 1 & 1 & 0 & 0 & 1 & 2 & 0 & 0 & 0 & 0\end{array}\right]$

First offspring

$\left[\begin{array}{lllllllllll}1 & 1 & 1 & 0 & 0 & 1 & 1 & 0 & 0 & 1 & 2 \\ 1 & 0 & 0 & 1 & 2 & 1 & 2 & 1 & 2 & 0 & 0 \\ 2 & 0 & 0 & 0 & 0 & 1 & 1 & 1 & 1 & 1 & 2 \\ 3 & 1 & 3 & 0 & 0 & 1 & 2 & 1 & 2 & 0 & 0\end{array}\right]$

For the string part of the chromosome, a random number is selected between 2 and length of string minus 1 . This number divides selected parents into two parts. Combination of these parts generates two offspring. First part of first parent along with second part of second parent make offspring one and second part of first parent along with first part of second parent make offspring two. Following with the operation, modifications are done and repetitive members replace missing ones. An example is presented below to clarify the operation.

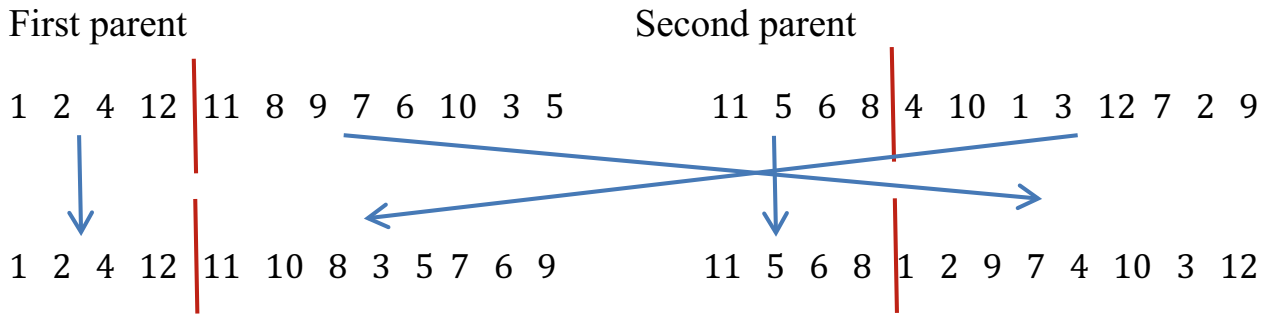

Second parent

$\left[\begin{array}{lllllllllll}2 & 1 & 1 & 1 & 1 & 1 & 1 & 0 & 0 & 0 & 0 \\ 1 & 0 & 0 & 1 & 2 & 1 & 2 & 1 & 2 & 0 & 0 \\ 3 & 0 & 0 & 1 & 3 & 1 & 1 & 1 & 3 & 0 & 0 \\ 3 & 1 & 3 & 0 & 0 & 1 & 2 & 1 & 2 & 0 & 0\end{array}\right]$

\section{Second offspring}

$\left[\begin{array}{lllllllllll}2 & 1 & 1 & 1 & 1 & 1 & 1 & 0 & 0 & 0 & 0 \\ 2 & 1 & 1 & 1 & 3 & 0 & 0 & 1 & 3 & 0 & 0 \\ 3 & 0 & 0 & 1 & 3 & 1 & 1 & 1 & 3 & 0 & 0 \\ 2 & 1 & 1 & 0 & 0 & 1 & 2 & 0 & 0 & 0 & 0\end{array}\right]$

Note that, for both crossover operations, the operator works only in $50 \%$ of the time. So, under this condition, offspring with changes in just one part of the chromosome are possible.

\section{Mutation operator}

In the matrix part of the chromosome, mutation operator performs as follows. Based on mutation rate, number of rows is selected randomly and for each selected row, a new route generates using of same process in initiating the population. In the next step, selected and primary routes (rows) distances are computed and compared together. If the new route has less distance, it replaces in the related row, otherwise primary row reserves.

For the string part of the chromosome, mutation operator is randomly selected from a set of five different mutation functions. In the first function, two different members of the string are randomly selected to be replaced with each other. 


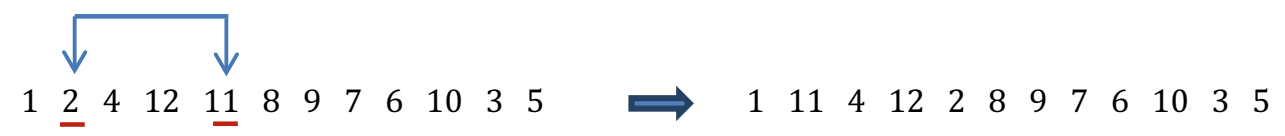

In second function, in addition to replacing the members, the order of members between them is reversed.

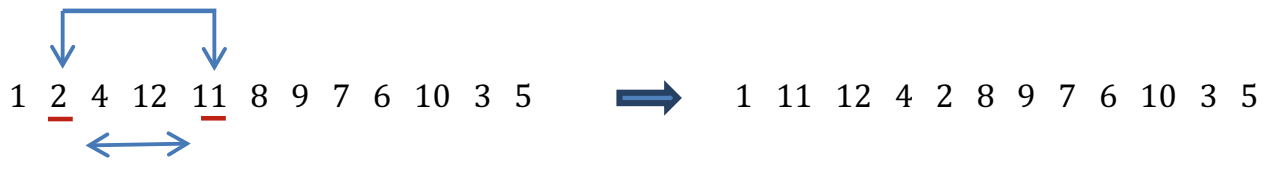

In third function, two members are random taken and the first member is moved after the second member.

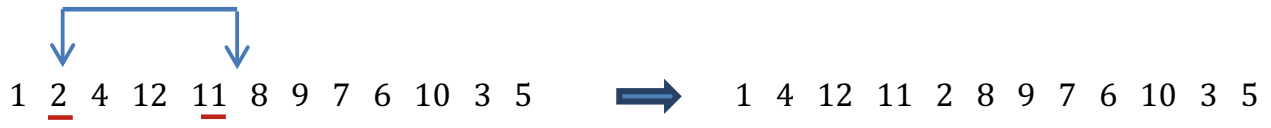

In the fourth function, the assigned retailers for each tour are determined. Then, their order is reversed in the routes.

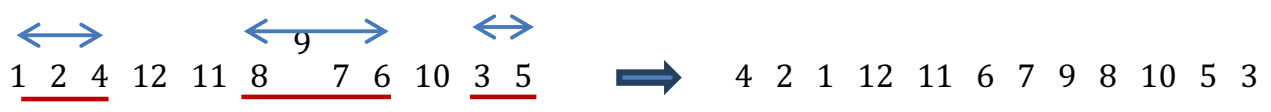

In the fifth function, after determining the retailer tours, two tours are selected randomly and a random member of each selected tour is exchanged.

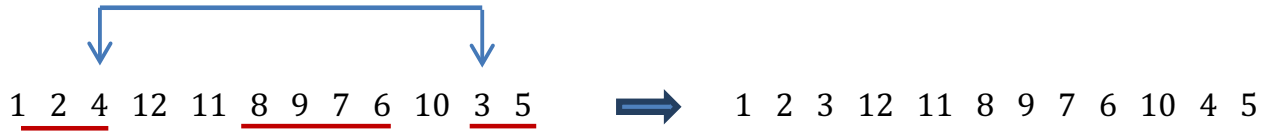

Similar to crossover operator, mutation operators apply at a probability of $50 \%$. As mentioned, this allows for the generation of offspring wherein only one part of the chromosome is changed.

\section{Fitness function}

The model's objective function is used as the fitness function. For each matrix part of the chromosome, a string part is generated; coupled together, both parts are used as a single chromosome in fitness function. For each chromosome, four cost functions and one penalty function are developed. The first cost function addresses the cost of routing tours from DCs to retailers. As explained before, open DCs and their allocated tours are determined based on the string part of the chromosome. Then, DC numbers are added to the beginning and end of each related tour before determining the distance between each pair of nodes along the tour. Lastly, overall tour cost can obtain by summing up the calculated distances and multiplying the result by the product's unit transportation cost.

In second cost function, established DCs are distinguished using string part of the chromosome and summation of their establishment costs is calculated.

To determine transportation cost on the multimodal network (from supplier to potential DCs), the following process is applied. As a first step, the matrix part of the chromosome is decomposed into its rows and in each row 
the intermediate nodes used along the route are identified. In the second step, transportation mode between nodes is distinguished. In third step, distances between nodes are determined and multiplied by the corresponding transportation cost; sum of the results gives total cost for each link. Finally, total demand for each DC is calculated and multiplied by link costs. Sum of these costs form the multimodal network transportation cost. Last cost function is the cost of providing mode changing facilities at multimodal terminals. To have the cost calculated, nodes which mode changes are happened should be identified. For this mean, inbound and outbound modes are determined for each node along multimodal links. If the modes are identical, then node may host no mode change; otherwise mode changing facilities need to be established on that location. Fixed cost of providing mode changing facilities is aggregated to form the fourth cost function.

For this fitness function, a penalty is considered for violating vehicle capacity along the routing tours. If sum of assigned retailers' demands to a potential DC violated corresponding vehicle capacity, the penalty is applied.

\section{Forming the next population}

To form the next generation, first population along with offsprings generated by crossover operator and mutants are sorted on the basis of their costs. New population with the same size as first population is selected from the first members of the set (fewer costs are selected).

\section{Stop condition}

The algorithm is set to stop once a predefined maximum number of iterations is achieved.

\section{Algorithm parameter setting}

Parameter setting is an important part of coding the algorithm where different parameter configurations contribute to the quality of answers. In this study, running the algorithm with different parameter configuration arrived to following optimum values of GA parameters (Table 1).

Table 1 Genetic algorithm parameters

\begin{tabular}{llll}
\hline Parameter name & Value & Parameter name & Value \\
\hline Population size & 400 & Roulette wheel selection pressure & 20 \\
Mutation rate & 0.8 & Mutation implementation rate & 0.8 \\
Crossover rate & 0.8 & & \\
\hline
\end{tabular}

\section{Numerical cases and analysis of the results}

In this section, two numerical cases were generated and solved. Proposed genetic algorithm and CPLEX solver were used to solve the cases. GA coded in MATLAB software and GAMS software was used to run CPLEX software and obtained results compared. A personal laptop equipped with an Intel ${ }^{\circledR}$ Core $^{\mathrm{TM}}$ i3 $\mathrm{CPU}$ at $2.4 \mathrm{GHz}$ and $6 \mathrm{~GB}$ of RAM was used to process the data. Also, different cost scenarios were designed to analyze model and algorithm sensitivity.

\section{Case 1: description}

Each numerical case have two parts; a multimodal network along with a single-mode network of DCs and retailers. In case 1 , the multimodal network is generated by random data. It consists of 1 supplier, 8 intermediate nodes, 2 potential DCs, 15 road links, 19 railway links and 14 seaway links. The distances between nodes along the road network were uniformly generated in $[0,400]$ interval (First $x$ and $y$ coordination generated for each node, then Euclidean distances calculated). To generate railway network distances, another uniform distribution over interval $[0,20]$ is used, and results added to the Euclidean distances. Adding another random number to railway network distances from interval $[0,20]$, generates seaway network distances. Table 2 shows the defined links and their distances along the three transportation networks.

For second part of the numerical case 1, five retailers were considered. Table 3 shows their $\mathrm{x}$ and $\mathrm{y}$ coordination and demands. This part of the numerical case extracted from LRP instances with 20 customers and 5 DCs reported by Prodhon (2010). Establishment costs of DC 1 and DC 2 were 10,841 and 11,961 , respectively, with a unit on-vehicle transportation cost of 20.

Table 4 displays GAMS and GA results for case 1 under different cost and capacity scenarios. Scenarios created by making changes in transportation cost along road, rail and sea networks, DCs' capacity and mode changing cost. As mentioned before, mode changing cost refers to the cost of providing facilities at multimodal terminals where transportation modes can be changed. It should be note that constant parameter $B$ ( $B$ is a large constant parameter used in constraints 3 and 6 ) determined by solving first scenario for different values and finally set to 100,000 .

All best multimodal paths start from node 1 (supplier) and pass intermediate nodes to reach DCs. Notations $H, R$, and $S$ between the nodes refer to road, railway, and seaway modes. Best routes start from DCs and pass through retailers (retailers numbers are different from multimodal 
Table 2 Multimodal network for the three numerical examples
Table 3 DCs, retailers coordinates, and demands for the case 1

\begin{tabular}{llllllllll}
\hline Node number & Exiting arc & Road & Rail & Sea & Node number & Exiting arc & Road & Rail & Sea \\
\hline 1 & $(1,2)$ & 23 & 27 & - & 4 & $(4,6)$ & 184 & - & 204 \\
1 & $(1,3)$ & - & 87 & - & 4 & $(4,7)$ & - & 136 & 146 \\
1 & $(1,4)$ & - & 197 & - & 4 & $(4,8)$ & - & 373 & 390 \\
1 & $(1,5)$ & 331 & - & - & 5 & $(5,7)$ & 170 & 179 & 188 \\
2 & $(2,3)$ & 60 & - & 70 & 5 & $(5,8)$ & 304 & 315 & 360 \\
2 & $(2,4)$ & - & 179 & - & 6 & $(6,8)$ & 214 & 220 & 227 \\
2 & $(2,5)$ & - & 300 & 313 & 6 & $(6, \mathrm{DC} 1)$ & 287 & 300 & 315 \\
2 & $(2,7)$ & 350 & 383 & - & 7 & $(7,8)$ & 190 & 197 & 310 \\
3 & $(3,4)$ & 100 & 111 & - & 7 & $(7, \mathrm{DC} 1)$ & 259 & 260 & 268 \\
3 & $(3,6)$ & - & 275 & 282 & 7 & $(7, \mathrm{DC} 2)$ & 332 & 343 & 357 \\
3 & $(3,7)$ & - & 320 & - & 8 & $(8, \mathrm{DC} 2)$ & 155 & 164 & 177 \\
4 & $(4,5)$ & 134 & - & - & & & & & \\
\hline
\end{tabular}

\begin{tabular}{llllllll}
\hline Coordinates & DC 1 & DC 2 & Retailer 1 & Retailer 2 & Retailer 3 & Retailer 4 & Retailer 5 \\
\hline$X$ & 6 & 19 & 20 & 8 & 29 & 18 & 19 \\
$Y$ & 7 & 44 & 35 & 31 & 43 & 39 & 47 \\
Demand & - & - & 18 & 13 & 19 & 12 & 18 \\
\hline
\end{tabular}

path numbers) and eventually returning back to the original DC.

Under scenario 1, mode changing cost (cost of providing mode changing facilities at multimodal terminals) is much lower than DC establishment cost (about one hundredth). Furthermore, transportation cost on road and railway networks, compare to seaway network, are triple and twice, respectively; and considered capacity for each DC is adequate to satisfy all retailers' demands. Result show that under this scenario, only DC1 (with lower establishment cost) established. Because of high DC establishment cost, when one DC can answer to all the retailers demand (high vehicle capacity), just one DC establishes. In this scenario, seaway has the lowest cost and mode changing cost is negligible. So multimodal route is consists of four seaway and one railway links and multimodal terminal established at node 2. Under scenario 2, mode changing cost is raised to 10,000 (close to that of DC establishment cost). However, no change is seen in the results, i.e., seaway transportation still presents a costly justifiable approach, and similar to scenario 1 , a multimodal terminal established at node 2 . In scenario 3 , mode changing cost is about five times larger than DC establishment cost. The results show that change in transportation mode is no longer economic, and multimodal route products transported from supplier to potential DC 1 along the railway network. With sufficient vehicle capacity for all the demands, only one DC is established. Under scenario 4, mode changing cost set to 10,000 and vehicle capacity decreased to 70 , so that a single DC cannot answer all the demands. In this scenario, both of DCs established with a different multimodal route and delivery tour for each DC. In scenario 5, differences between transportation costs for three modes are lower than previous scenarios. Again, mode changing cost and vehicle capacity are set to 10,000 and 70 , respectively. Results show that in the multimodal network, products are transport along road (due to lower distance) and seaway (due to lower cost) networks. Since DC capacity has not changed, retailer routes are the same. Sixth scenario is similar to previous scenario with raise in mode changing cost. In this scenario model, choose a multimodal route with no changing in modes, so railway network (due to its continuity from the supplier to DCs) is preferred over road network (with lower transportation distances) and seaway network (with lower costs). The results show that a change in vehicle capacity can change the number of established DCs and affect routs to retailers.

These scenarios are further solved by genetic algorithm and the results reported in Table 4. Results show that all runs of the algorithm reached optimum solutions which normally achieved between iterations 5 and 15 . The results demonstrated that GA provides proper performance and solve problem in a reasonable run time.

\section{Case 2}

Example generated by Xiong and Wang (2012) used in case 2 , for multimodal part of the problem. The authors defined a multimodal network with 35 nodes and three modes including truck, rail and barges transportation. Five 
Table 4 Results of case 1 under different scenarios

\begin{tabular}{|c|c|c|c|c|c|c|c|c|c|c|c|c|}
\hline \multirow[t]{2}{*}{$\begin{array}{l}\text { Scenario } \\
\text { number }\end{array}$} & \multicolumn{3}{|c|}{$\begin{array}{l}\text { Shipment cost for } \\
\text { each mode }\end{array}$} & \multirow{2}{*}{$\begin{array}{l}\text { Transfer } \\
\text { cost } \\
\text { between } \\
\text { modes }\end{array}$} & \multirow[t]{2}{*}{$\begin{array}{l}\text { Vehicle } \\
\text { capacity }\end{array}$} & \multirow[t]{2}{*}{$\begin{array}{l}\text { GAMS } \\
\text { value }\end{array}$} & \multirow{2}{*}{$\begin{array}{l}\text { GAMS } \\
\text { solution } \\
\text { time (s) }\end{array}$} & \multicolumn{3}{|l|}{ GA values } & \multirow{2}{*}{$\begin{array}{l}\text { Best } \\
\text { multimodal } \\
\text { path }\end{array}$} & \multirow[t]{2}{*}{$\begin{array}{l}\text { Best } \\
\text { route }\end{array}$} \\
\hline & Road & Rail & Sea & & & & & Worst & Medium & Best & & \\
\hline 1 & 3 & 2 & 1 & 100 & 100 & 64,061 & 1.06 & 64,061 & 64,061 & 64,061 & $\begin{array}{c}\text { 1-R-2-S-3- } \\
\text { S-4-S-7- } \\
\text { S-DC1 }{ }^{\text {a }}\end{array}$ & $\begin{array}{l}\text { DC1-2- } \\
1-4-5- \\
3- \\
\text { DC1 }^{b}\end{array}$ \\
\hline 2 & 3 & 2 & 1 & 10,000 & 100 & 73,961 & 0.59 & 73,961 & 73,961 & 73,961 & $\begin{array}{c}\text { 1-R-2-S-3- } \\
\text { S-6-S-8- } \\
\text { S-DC1 }\end{array}$ & $\begin{array}{c}\text { DC1-2- } \\
1-4-5- \\
3-D C 1\end{array}$ \\
\hline 3 & 3 & 2 & 1 & 50,000 & 100 & 107,801 & 0.64 & 107,801 & 107,801 & 107,801 & $\begin{array}{l}\text { 1-R-4-R-7- } \\
\text { R-DC1 }\end{array}$ & $\begin{array}{c}\text { DC1-2- } \\
1-4-5- \\
3-D C 1\end{array}$ \\
\hline \multirow[t]{2}{*}{4} & 3 & 2 & 1 & 10,000 & 70 & 87,270 & 1.08 & 87,270 & 87,270 & 87,270 & $\begin{array}{c}\text { 1-R-2-S-3- } \\
\text { S-4-S-7- } \\
\text { S-DC1 }\end{array}$ & $\begin{array}{c}\text { DC1-2- } \\
1-5-3- \\
\text { DC1 }\end{array}$ \\
\hline & & & & & & & & & & & $\begin{array}{c}\text { 1-R-2-S-3- } \\
\text { S-4-S-7- } \\
\text { S-DC2 }\end{array}$ & $\begin{array}{l}\text { DC2-4- } \\
\text { DC2 }\end{array}$ \\
\hline \multirow[t]{2}{*}{5} & 23 & 22 & 21 & 10,000 & 70 & $1,073,830$ & 1.42 & $1,073,830$ & $1,073,830$ & $1,073,830$ & $\begin{array}{c}\text { 1-H-2-H-3- } \\
\text { S-4-S-7- } \\
\text { S-DC1 }\end{array}$ & $\begin{array}{c}\text { DC1-2- } \\
1-5-3- \\
\text { DC1 }\end{array}$ \\
\hline & & & & & & & & & & & $\begin{array}{c}\text { 1-H-2-H-3- } \\
\text { S-4-S-7- } \\
\text { S-DC2 }\end{array}$ & $\begin{array}{c}\text { DC2-4- } \\
\text { DC2 }\end{array}$ \\
\hline \multirow[t]{2}{*}{6} & 23 & 22 & 21 & 50,000 & 70 & $1,090,753$ & 1.13 & $1,090,753$ & $1,090,753$ & $1,090,753$ & $\begin{array}{l}\text { 1-R-4-R-7- } \\
\text { R-DC1 }\end{array}$ & $\begin{array}{c}\text { DC1-2- } \\
1-5-3- \\
\text { DC1 }\end{array}$ \\
\hline & & & & & & & & & & & $\begin{array}{l}\text { 1-R-4-R-7- } \\
\text { R-DC2 }\end{array}$ & $\begin{array}{l}\text { DC2-4- } \\
\text { DC2 }\end{array}$ \\
\hline
\end{tabular}

${ }^{a}$ Multimodal route starts from supplier (node 1) and paths nodes number 2, 3, 4, 7 and end to DC1. Transportation links between supplier and node number 2 is a railway $(\mathrm{R})$ and other transportation links are seaway (S)

${ }^{\mathrm{b}}$ Retailers delivery tour starts from DC1 and meets retailers number 2, 1, 4, 5 and 3, respectively, and returns to DC1

ending nodes (nodes $31-35$ ) considered as potential DCs and node 1 defined as supplier location.

Some modifications did on multimodal network. According to the problem assumptions, all links between potential DCs deleted. As a result, node 34 (potential DC 4) lose all of its communications to other nodes. To modify that three truck, rail and barge links from node 27-34 added to multimodal network. Also, every defined distance for multimodal network is multiplied by 10 to provide more consistency with DCs and retailers distances. For LRP part of the problem, Prodhon (2006) instance with 5 potential DCs and 20 customers used (Prodhon 2010).

Software and algorithm results for case 2 under different cost and capacity scenarios are presented in Table 5. Also, for this case, the parameter $B$ is set to the same value $(100,000)$.

Same scenarios defined and solved for case 2. However, in contrary to the case 1 , cost of a change in transportation mode had lower impact on changing multimodal routes. This is because of significant differences between distances of transportation modes. Compared to other modes, road network has lower distances, so under these cost scenarios, using a road network is costly justifiable, even with higher transportation cost.

In the first three scenarios capacity of DCs was set to 300; so two DCs could adequately answer all of the demands. The results show that under all three scenarios, DCs 3 and 4 which had lower establishment cost, selected to establish. Under scenarios 1 and 3, mode changing cost is low, so the node 17 selected for multimodal terminal. Under scenario 2, mode changing cost is raised to 10,000 ; such a high cost caused a change in multimodal route to avoid mode change; however, route structure changes very slightly.

Under scenarios 4, 5 and 6, DCs' capacity was 70; as a result, all the DCs should be established to fulfill all the 


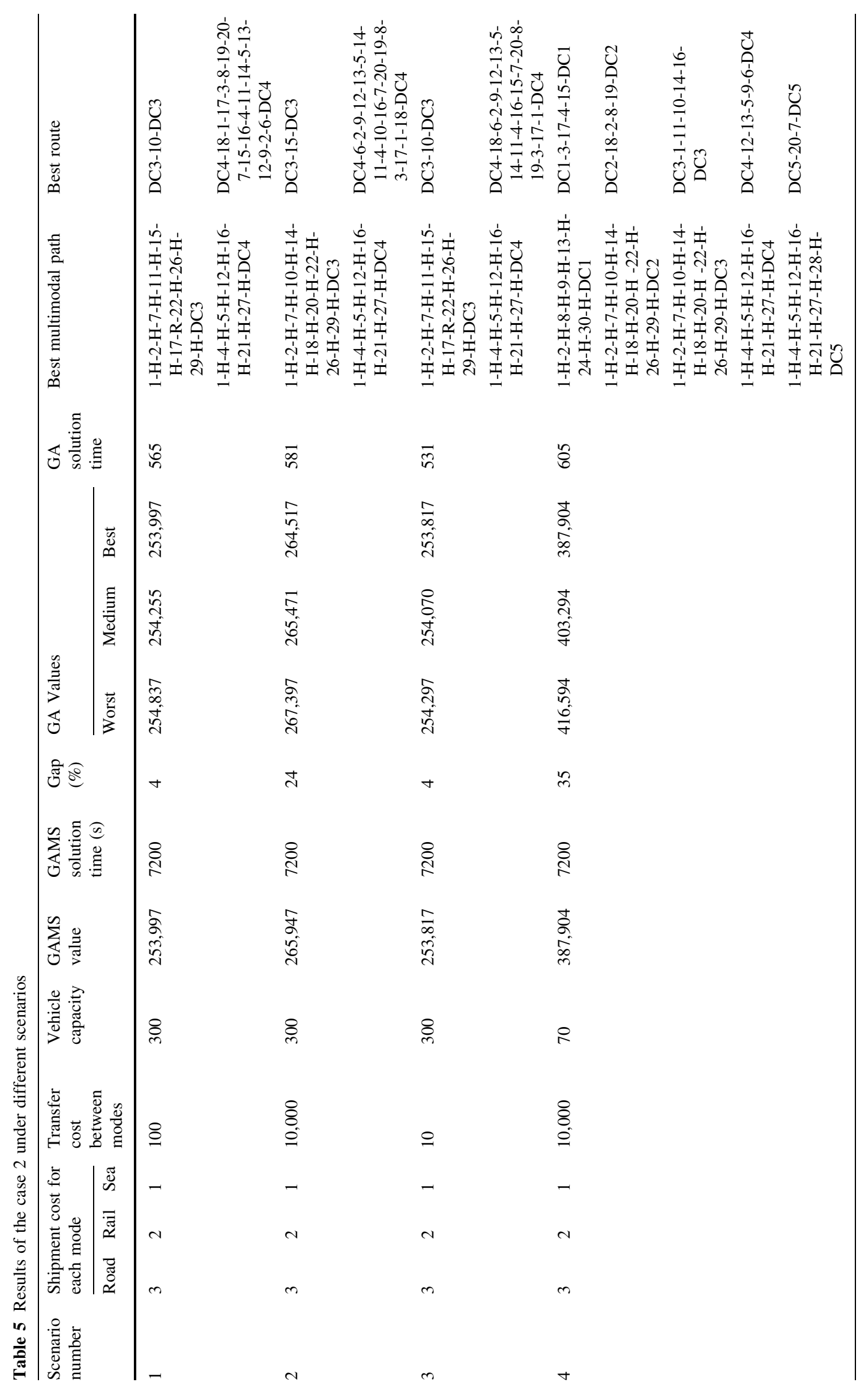




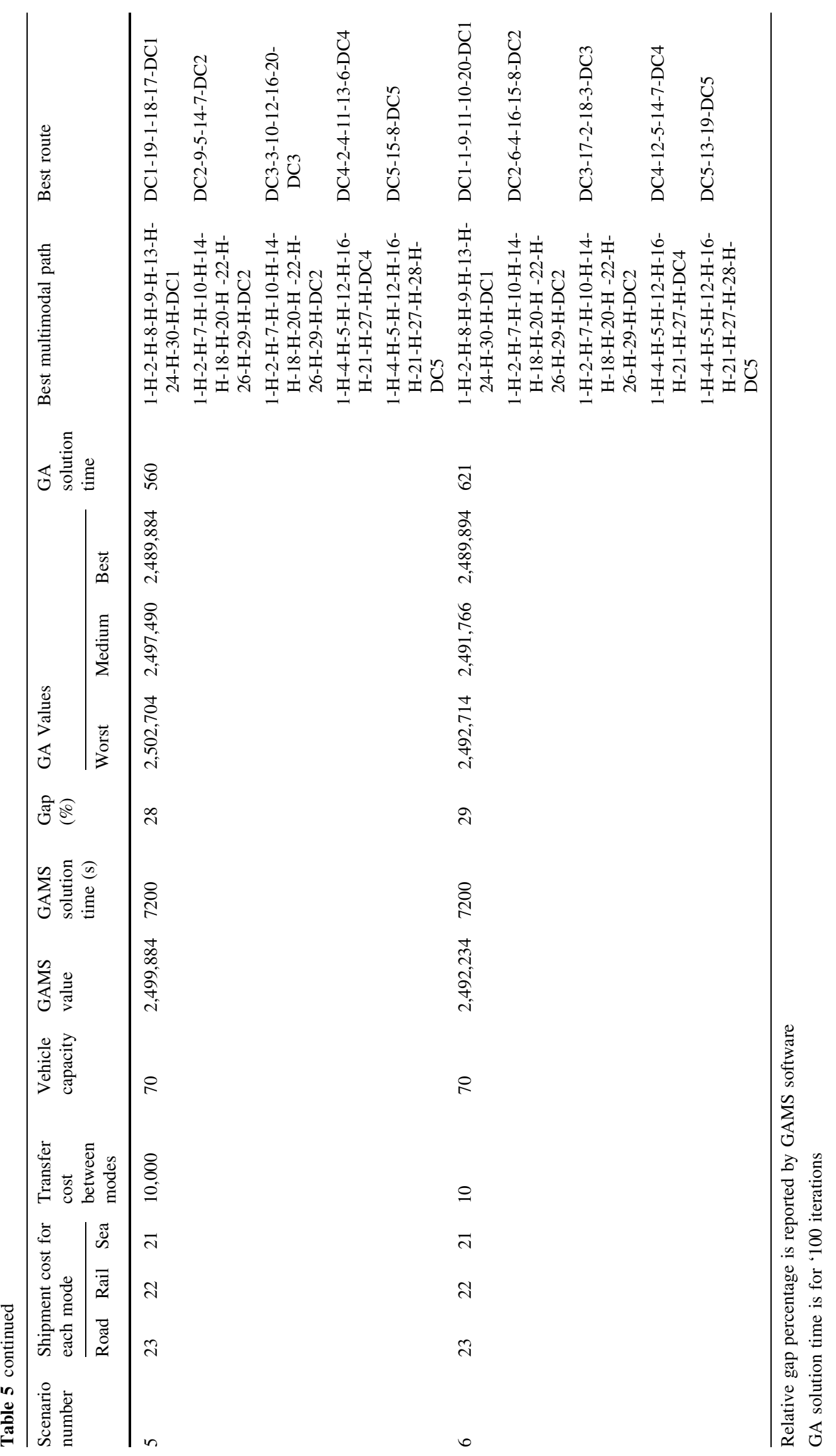


demands. In scenario 5, transportation costs were all close together compared to scenario 4 , however, such a change had no impact on multimodal route. So in this case, transportation distance is more important than transportation cost and road transportation is the popular mode due to its lower distances. Under scenario 6, mode changing cost was 10 , but yet no change was evident on multimodal route. Comparing results with scenario 3 reveals that with the same mode changing cost, a different route was attained for DC 3. In fact, when rail and road modes costs were 2 and 3, respectively, transportation mode was likely to change at node 17 , but when the costs were 22 and 23 , respectively, this was not the case and transportation continued along the road network.

For the same established DCs, different tours from DC to retailers were attained under different scenarios. For example, under scenarios 1 and 2, different tours were attained for DC 3 and DC 4. However, according to the same transportation cost and identical distances between retailers and DC, such a difference was not expected (similar to case 1). The situation was attributed to suboptimality of the solutions given by the software under different scenarios. GAMS reported a feasible solution within limited run time. GA reported an equal or even better solution with lower run times, but the optimality of the answers is yet to be proved.

Although the case 2 was considered as a medium-size problem, because of high complexity of the model and NPhard nature of the problem (Shen et al. 2003), GAMS failed to find an optimal solution within the limited run time. Existing gap within the solutions are reported in Table 5.

Comparing GA and GAMS results indicate that for three scenarios results are identical but for the other three scenarios, GA gave better solutions. Also, GA run times for 100 iterations are reported in Table 5, showing that the GA tends to gives a proper solution within a reasonable time, making the algorithm useful for large-size cases.

\section{Conclusions}

As a new branch of location problems, location-routing problem is still under development by various researchers. Present paper considered a location-routing problem on multimodal network. This paper aims to model and solve two problems at the same time. First problem is to select multimodal routes from supplier to potential DCs along with locating multimodal terminals. Second problem is DC location with routing tours from located DCs to retailers. An integer linear programming proposed and a genetic algorithm developed to capture the problem structure. To validate mathematical model, analyze sensitivity and demonstrate algorithm performance, two small and large size numerical instances generated based on previous papers, and different cost scenarios applied to these instances. Scenarios were different in vehicle capacity, transportation modes' costs, and mode changing cost. According to the results, for different scenarios, different multimodal routes selected. Changing mode change cost affects on establishing multimodal terminals. High mode changing cost caused products to be transported on just one transportation mode; decreasing the cost, however, led to changes in modes, requiring multimodal terminals to be established. Changing vehicle capacity cause a change in number of established DCs and retailers orders on delivery tours. Also, by changing transportation cost on multimodal network, model makes a trade-off between distance and transportation cost and selects a multimodal route with lowest cost. In large numerical instance, due to high complexity of the model, GAMS software failed to find an optimum solution within a reasonable time. In this case, genetic algorithm run under different scenarios and ended up returning solutions equal to or better than GAMS results, revealing good performance of the algorithm.

For future researches, first suggestion is to develop other solving algorithms including exact algorithms and comparing results. Other developments to LRP can be other suggestion for future research; mathematical model can be further developed considering uncertainties within data and dynamic programming issues, for example. Applying model for real cases and reporting real results can be another validation for model.

Open Access This article is distributed under the terms of the Creative Commons Attribution 4.0 International License (http://crea tivecommons.org/licenses/by/4.0/), which permits unrestricted use, distribution, and reproduction in any medium, provided you give appropriate credit to the original author(s) and the source, provide a link to the Creative Commons license, and indicate if changes were made.

\section{References}

Afshar-Bakeshloo M, Mehrabi A, Safari H, Maleki M, Jolai F (2016) A green vehicle routing problem with customer satisfaction criteria. J Ind Eng Int 12(4):529-544

Aghighi A, Malmir B (2016) Designing distribution networks of perishable products under stochastic demands and routs. In: International conference on industrial engineering and operations management, Sep, Detroit, Michigan

Ahmadi-Javid A, Seddighi A (2013) A location-routing problem with disruption risk. Transp Res Part E Logist Transp Rev 53:63-82

Albareda-Sambola M, Fernández E, Nickel S (2012) Multi period location-routing with decoupled time scales. Eur J Oper Res 217:248-258

Alumur S, Kara B, Karasan O (2012) Multimodal hub location and hub network design. Omega 40:927-939

Ayar B, Yaman H (2012) An intermodal multi-commodity routing problem with scheduled services. Comput Optim Appl 53:131-153 
Azadeh A, Elahi S, Hosseinabadi Farahani M, Nasirian B (2017) A genetic algorithm-Taguchi based approach to inventory routing problem of a single perishable product with transshipment. Comput Ind Eng 104:124-133

Bontekoning YM, Macharis C, Trip JJ (2004) Is a new applied transportation research field emerging? A review of intermodal rail-truck freight transport literature. Transp Res Part A 38:1-34

Button KJ (1994) Transport economics. University Press, Cambridge

Chiadamrong N, Kawtummacha R (2008) A methodology to support decision-making on sugar distribution for export channel: a case study of Thai sugar industry. Comput Electron Agric 64:248-261

Chopra S, Meindl P (2001) Supply chain management: strategy, planning, and operations. Prentice-Hall, Upper Saddle River

Crainic T (2003) Long-haul freight transportation. In: Hall RW (ed) Handbook of transportation science. International series in operations research and management science, vol 56. Springer, US, pp 451-516

Daskin MS, Synder LV, Berger RT (2005) Facility location in supply chain design. In: Langevin A, Riopel D (eds) Logistics systems: design and optimization. Springer, Berlin

Drexl M, Schneider M (2014) A survey of variants and extensions of the location-routing problem. Eur J Oper 241(2):283-308

Fazel Zarandi MH, Hemmati A, Davari S, Turksen B (2013) Capacitated location-routing problem with time windows under uncertainty. Knowl Based Syst 37:480-489

Gao Sh, Wang Y, Cheng J, Inazumi Y, Tang Zh (2016) Ant colony optimization with clustering for solving the dynamic location routing problem. Appl Math Comput 285:149-173

Ghezavati VR, Beigi M (2016) Solving a bi-objective mathematical model for location-routing problem with time windows in multiechelon reverse logistics using metaheuristic procedure. J Ind Eng Int 12(4):469-483

Govindan K, Jafarian A, Khodaverdi R, Devika K (2014) Two echelon multiple vehicle location-routing problem with time windows for optimization of sustainable supply chain network of perishable food. Int J Prod Econ 152:9-28

Hajibabai L, Ouyang Y (2013) Integrated planning of supply chain networks and multimodal transportation infrastructure expansion: model development and application to the biofuel industry. Comput Aided Civ Infrastruct Eng 28:247-259

Hiassat A, Diabat A, Rahwan I (2017) A genetic algorithm approach for location-inventory-routing problem with perishable products. J Manuf Syst 42:93-103

Karaoglan I, Altiparmak F, Kara I, Dengiz B (2012) The locationrouting problem with simultaneous pickup and delivery: formulations and a heuristic approach. Omega 40:465-477

Laporte G, Nobert Y (1981) An exact algorithm for minimizing routing and operating costs in depot location. Eur J Oper Res 6:224-226

Laporte G, Nobert Y, Pelletier J (1983) Hamiltonian location problems. Eur J Oper Res 12:82-89

Li ZC, Hung HGJ, Lam WK, Wang SC (2007) A Model for evaluation of transport policies in multimodal networks with road and parking capacity constraints. J Math Model Algorithm 6:239-257 (Springer)

Lopes RB, Ferreira C, Santos BS, Barreto S (2013) A taxonomical analysis, current methods and objectives on location-routing problems. Int Trans Oper Res 20:795-822
Moccia L, Cordeau J, Laporte G, Ropke S, Valentini M (2010) Modeling and solving a multimodal transportation problem with flexible time and scheduled services. Networks 57:53-68

Nagy G, Salhi S (2007) Location-routing: issues, models and methods. Eur J Oper Res 177:649-672

Najjartabar M, Shetaban S, Malmir B (2016) An Integrated locationinventory model for supply chain network with correlated demand. In: Proceedings of the 2016 international conference on information systems, logistics and supply chain, June 1-4, Bordeaux, France

Najjartabar-Bisheh M, Delavari M, Malmir B (2017) Role of thirdparty companies in a sustainable supply chain design. Int $\mathbf{J}$ Logist Syst Manag (in press)

OECD (1997) Road transport research, outlook 2000. OECD, Paris

Prodhon C (2010) http://prodhonc.free.fr/Instances/instances_us.htm. Accessed 30 June 2017

Prodhon C, Prins C (2014) A survey of recent research on locationrouting problems. Eur J Oper Res 238(1):1-17

Riquelme-Rodríguez JP, Gamache M, Langevin A (2016) Location arc routing problem with inventory constraints. Comput Oper Res 76:84-94

Rodriguez-Martin I, Salazar-Gonzalez JJ (2014) A branch-and-cut algorithm for the hub location and routing problem. Comput Oper Res 50:161-174

Samanlioglu F (2013) A multi-objective mathematical model for the industrial hazardous waste location-routing problem. Eur J Oper Res 226:332-340

Seyedhosseini SM, Makui A, Shahanaghi K, Torkestani SS (2016) Models, solution, methods and their applicability of dynamic location problems (DLPs) (a gap analysis for further research). J Ind Eng Int 12(3):311-341

Shahabi M, Unnikrishnan A, Jafari-Shirazi E, Boyles SD (2014) A three level location-inventory problem with correlated demand. Transp Res Part B 69:1-18

Shen ZM, Coullard C, Daskin MS (2003) A joint location-inventory model. Transp Sci 1(37):40-55

SteadieSeifi M, Dellaert NP, Nuijten W, Van Woensel T, Raoufi R (2014) Multimodal freight transportation planning: a literature review. Eur J Oper Res 233:1-15

Tavakkoli-Moghaddam R, Raziei Z (2016) A location-routing problem with disruption risk. Transp Res Part E Logist Transp Rev 53:63-82

Tiwari MK, Kumar RA, Mohapatra P, Yew WK, Benyoucef L (2013) Route selection and consolidation in international intermodal freight transportation. In: Benyoucef L et al (eds) Applications of multi-criteria and game theory approaches. Springer Series in Advanced Manufacturing, Berlin, pp 181-194

Tuzkaya UR, Onut S, Tuzkaya G (2014) A Strategic planning methodology for the multimodal transportation systems: a case study from Turkey. J Appl Math ID 931456:23

Wu X, Lei N, Meng X (2017) Designing an integrated distribution system for catering services for high-speed railways: a threeechelon location routing model with tight time windows and time deadlines. Transp Res Part C 74:212-244

Xie Y, Lu W, Wang W, Quadrifoglio L (2012) A multimodal location and routing model for hazardous materials transportation. J Hazard Mater 227-228:135-141

Xiong G, Wang Y (2012) Best routes selection in multimodal networks using multi-objective genetic algorithm. J Comb Optim. doi:10.1007/s10878-012-9574-8 (Springer) 